\title{
Ethnobotanical study of medicinal plants used by community in Jambur Labu Village, East Aceh, Indonesia
}

\author{
ELFRIDA, NINTA SABRINA TARIGAN, ADI BEJO SUWARDI ${ }^{\vee}$ \\ Department of Biology Education, Faculty of Teacher and Training Education, Universitas Samudra. Meurandeh, Kota Langsa 24416, Aceh, \\ Indonesia. Tel.: +62-641-426535. `email: adi.bsw@gmail.com
}

Manuscript received: 12 June 2021. Revision accepted: 24 June 2021.

\begin{abstract}
Elfrida, Tarigan NS, Suwardi AB. 2021. Ethnobotanical study of medicinal plants used by community in Jambur Labu Village, East Aceh, Indonesia. Biodiversitas 22: 2893-2900. Jambur Labu is a village in East Aceh that is home to various ethnic groups. The community in the village has used various plant species as a traditional medicine to treat diseases and disorders. The aim of this study was to investigate the medicinal plants used by the community in the Jambur Labu Village, Birem Bayeun Sub-district, East Aceh, Indonesia. This study was based on field surveys, plant collection, and interviews with the local people. Interviews were performed with 30 informants selected using the Snowball Sampling technique. A total of 107 medicinal plant species, belonging to 87 families, were documented to be used by the local community in Jambur Labu Village. Leaves were the most used plant part (38\%), followed by fruit $(22 \%)$, whole plant $(9 \%)$, rhizome $(8 \%)$, roots $(7 \%)$, flowers and bark (4\% each), sap (3\%), stem and tuber $(2 \%$ each), and seeds $(1 \%)$ and the main mode of preparations are decoction (39\%). Breynia androgyna (L.) Chakrab.dan N.P.Balakr., Moringa oleifera Lam, Jatropha multifida L., Zingiber montanum (J.Koenig) Link ex A.Dietr., Zingiber officinale Roscoe., and Tagetes erecta L. had the highest UV value (0.96), indicating the high importance of such species for the community. The high informant consensus factor $(\mathrm{ICF}=0.978)$ was assigned to the diseases of the blood and blood-forming organs and certain disorders involving the immune mechanism (DBF) category. The results of this study can enrich the existing study of medicinal uses of plant by communities, in this context is the use by a community in Aceh.
\end{abstract}

Keywords: Biodiversity, East Aceh, Jambur Labu, traditional medicine

\section{INTRODUCTION}

Indonesia is a mega-biodiversity country with over 30,000 flowering plant species, 6,000 of which are used for traditional healing processes (Elfahmi et al. 2014). However, only about $20-22 \%$ of them are cultivated, while the rest $78 \%$ are still harvested from the wild (Azmin et al. 2019). Local communities have long used various plant species for medicinal purposes (Jadid et al. 2020; Suwardi et al. 2021) as well as for food (Elfrida et al. 2020; Navia et al. 2015; Navia et al. 2020a; Suwardi et al. 2020a; Suwardi et al. 2020b; Navia et al. 2021), traditional ceremonies (Sutrisno et al. 2020), and household income (Suwardi et al. 2020c). The interaction between humans and plants has long been described as one of the factors influencing human civilization, particularly in medical fields (Yeung et al. 2020).

Ethnobotanical study is a method for investigating local and indigenous knowledge of certain communities regarding the use of plants to fulfill human needs including for medicinal purposes. Using ethnobotanical study, it is possible to investigate medicinal plants and how to use them as cultural characteristics in a specific community through these studies (Eldeen et al. 2016). Through the understanding of socio-cultural backgrounds, ethnobotanical information can provide valuable information for public health and environmental policies
(Weckmüller et al. 2019). Documenting plant medicinal uses through ethnobotanical studies allows for the development of modern drugs and treatments, and plant conservation (Calzada et al. 2020). The lack of documentation of medicinal plant use by local communities impedes the preservation of these plants (Rosita et al. 2007).

Many ethnobotanical studies in Indonesia describe the use of medicinal plants by the indigenous community for healing processes in which this knowledge has been practiced and inherited from generation to generation (Sujarwo et al. 2015; Batoro and Siswanto 2017; Mustofa et al. 2020; Rahmawati et al. 2020; Suwardi et al. 2021). In Indonesia, particularly in the Aceh province, there is a tendency of declining traditional knowledge among the younger generation (Navia et al. 2020b; Suwardi et al. 2020c). In addition, modernization has changed the lifestyle of the younger generation, resulting in a loss of local knowledge in certain communities (Weckmüller et al. 2019). As a result, efforts must be made to protect and maintain traditional knowledge, especially concerning the use of plants as traditional medicine. The aim of this study was therefore to investigate the medicinal plants used by the local community in Jambur Labu Village, Birem Bayeun Sub-district, East Aceh District, Aceh Province, Indonesia. 


\section{MATERIALS AND METHODS}

\section{Study period and area}

The study took place in Jambur Labu Village, Birem Bayeun Sub-district, East Aceh District, Aceh Province, Indonesia from March to May 2020 (Figure 1). Jambur Labu Village is located between $4^{\circ} 24^{\prime} 37.5^{\prime \prime} \mathrm{N}$, $97^{\circ} 55^{\prime} 08.8^{\prime}$ 'E with a total area of $7.11 \mathrm{~km}^{2}$, a hilly topography, and an elevation of $500 \mathrm{~m}$ asl. The climatic condition in Jambur Labu Village is tropical humid with average rainfall ranging from $346 \mathrm{~mm}$ to $3220 \mathrm{~mm}$ and a monthly temperature ranging from $26-30^{\circ} \mathrm{C}$. The Jambur Labu Village had a population of 2,289 people, with 1,188 men and 1,101 women. The Javanese and other tribes such as the Acehnese, Batak, and Minang are the dominant ethnic groups in this area (The Central Bureau of Statistics of East Aceh District 2020).

\section{Data collection procedure}

Data were obtained through semi-structured and structured interviews with informants who knew or used plants as medicine. Informant was selected using the Snowball Sampling technique by determining the key person (a figure that possesses strong power within society). The recommendation of the previous respondents determined the subsequent informants. In total, the interviews were conducted with 30 respondents to determine and explore the traditional knowledge regarding the use of medicinal plant species for the specific ailment(s), the utilized part(s), and the mode(s) of preparation or method(s) of processing the plants. The age of the informants ranged from 17 to more than 65 years (Table 1).

The majority of Jambur Labu Village's residents are Javanese. According to local people, their ancestors came from Java, where the Dutch East Indies government employed them in oil palm plantations decades ago. Regarding Indonesia's independence, they established settlements around the oil palm plantation area. Over time, their descendants began to convert forest into agricultural land and settled in the area, forming a village known as Jambur Labu. Other tribes, including Aceh, Minang, Gayo, and Batak, also settled in the village.

Plant samples were collected, as well as information about their vernacular names, the number of species, and their use. The identification of plant species was carried out at the Biology Laboratory, Samudra University, Aceh, Indonesia. The botanical names were crosschecked using Plants of the World online

(http://www.plantsoftheworldonline.org).

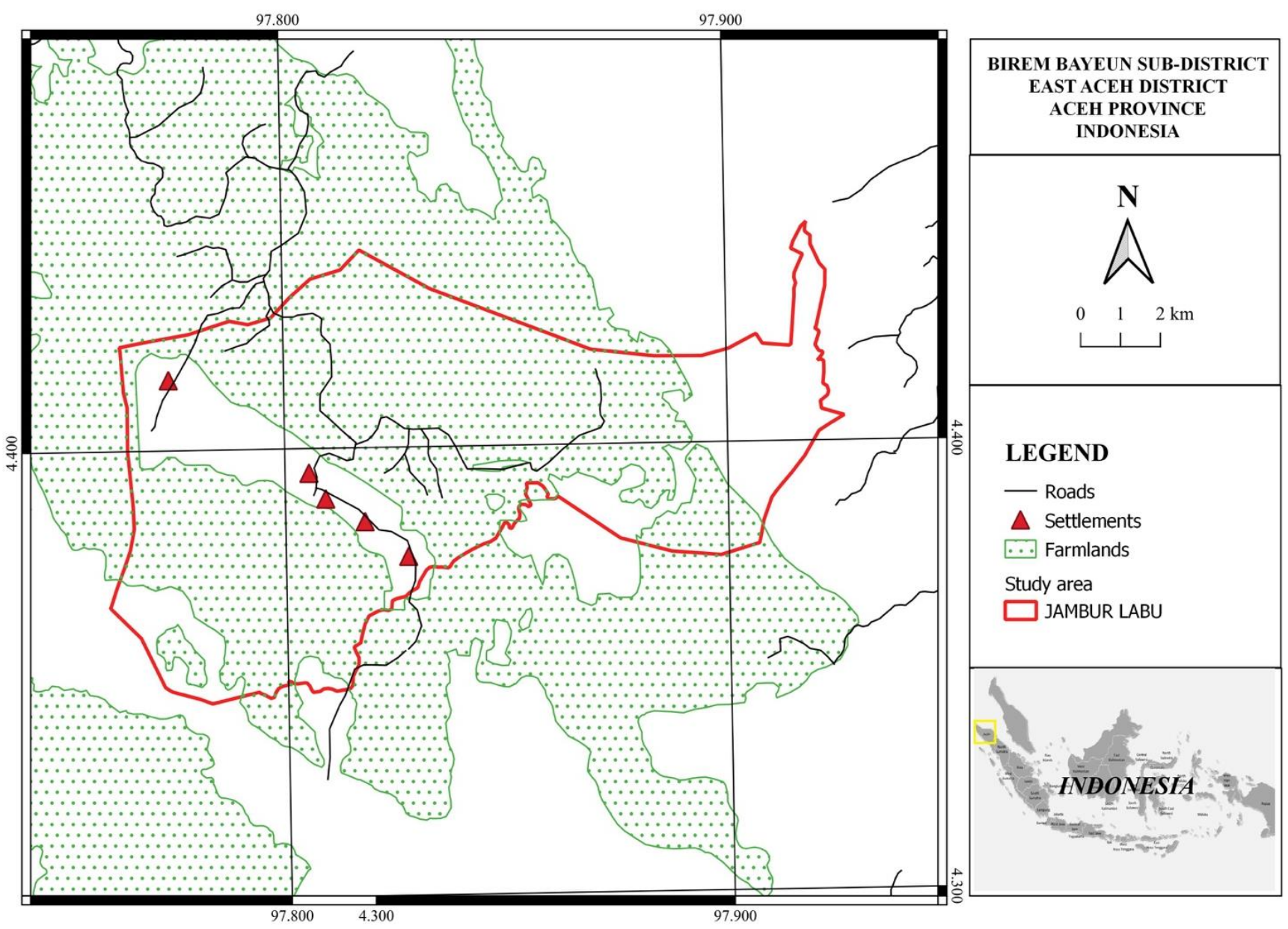

Figure 1. Map of the study area in Jambur Labu Village, Birem Bayeun Sub-district, East Aceh District, Aceh Province, Indonesia 
Table 1. The characteristics of the respondents Jambur Labu Village, Birem Bayeun Sub-district, East Aceh District, Aceh Province, Indonesia

\begin{tabular}{llcc}
\hline Parameters & Specification & Freq. & Percentage \\
\hline Gender & Male & 4 & 13.3 \\
& Female & 26 & 86.7 \\
Age (years) & $17-27$ & 5 & 16.7 \\
& $28-35$ & 9 & 30.0 \\
& $36-45$ & 7 & 23.3 \\
& $46-65$ & 7 & 23.3 \\
Education & $>65$ & 2 & 6.7 \\
& Elementary School & 19 & 63.3 \\
& Junior High School & 5 & 16.7 \\
Tribe & Senior High School & 4 & 13.3 \\
& University & 2 & 6.7 \\
& Javanese & 25 & 83.3 \\
& Aceh & 1 & 6.7 \\
& Minang & 1 & 3.3 \\
& Batak & 2 & 3.3 \\
& Gayo & 1 & 3.3 \\
\hline
\end{tabular}

\section{Data analysis}

Use Value (UV)

The Use Value was calculated as the ratio of the number of citations per species (U) to the number of informants $(\mathrm{N})$ using the following formula:

$$
\mathrm{UV}=\mathrm{U} / \mathrm{N}
$$

A plant with a high UV index has a high number of use reports, implying that it is important in the local community, while a low UV indicates that there have been few reports about its use.

\section{Informant Consensus Factor (ICF)}

Informant Consensus Factor was calculated using the formula:

$$
\mathrm{ICF}=(\mathrm{Nur}-\mathrm{Nt}) / \mathrm{Nur}-1
$$

Where: Nur defines the number of useful reports in each category, and $\mathrm{Nt}$ defines the total number of species used in a given category by all informants. The maximum value obtained in this Formula is 1, which indicates that the informants completely agree that the specific species cited could cure a specific ailment. A value of 0 , the minimum value, indicates that there is no exchange of information about plants among the informants.

\section{RESULTS AND DISCUSSION}

\section{Diversity of medicinal plants}

A total of 107 medicinal plant species were identified from the ethnobotanical survey in the study area, which was classified into 87 families and 52 genera (Table 2). The most represented family was Asteraceae with 8 species, followed by Euphorbiaceae ( 7 species), Rutaceae and Zingiberaceae (6 species each), Piperaceae (5 species), Myrtaceae (4 species), and Annonaceae and Rubiaceae (3 species each). The total species of medicinal plants found in this study (107 species) is higher than the medicinal plants found in the Subulussalam district, Aceh province (22 species) (Hasanuddin et al. 2021), and Kota Bahagia, South Aceh (96 species) (Suwardi et al. 2021). However, it is lower than the medicinal plants used by the sub-ethnic Batak Simalungun of North Sumatra (239 species) (Silalahi et al. 2015). The average number of species mentioned by each age category ranged from $10 \pm 5.1(17-27$ years old $)$ to $26.5 \pm 0.7$ (>65 years). Based on education level, the highest number of species was cited by respondents at the university level $(20.5 \pm 3.5)$, followed by junior high school $(15.2 \pm 8.1)$, elementary school $(13.5 \pm 6.5)$, and senior high school $(8 \pm 1.4)$.

\section{Plant part used}

The local community in the study area used different parts of the plants to treat various diseases. Leaves were the most used plant part (38\%), followed by fruit (22\%), whole plant $(9 \%)$, rhizome $(8 \%)$, roots $(7 \%)$, flowers and bark (4\% each), sap (3\%), stem and tuber (2\% each), and seeds (1\%) (Figure 2).

Local people in Jambur Labu Village used all parts of the plant from roots to leaves for medicinal uses. The part of medicinal plants mostly used by the community was the leaves and the least was the seeds. Several ethnobotanical surveys reported similar results with the leaves as the most frequently used plant parts (Malini et al. 2017; Suwardi et al. 2019; Navia et al. 2020b; Adam et al. 2020; Suwardi et al. 2021). Respondents preferred leaves as a raw material for traditional medicine because harvesting the leaves is less harmful to the plants. Leaves are also easy to collect and have good medicinal properties compared to other parts and do not depend on the season. The use of leaves as ingredients for medicinal herbs is considered an easier way of processing than the bark, stems, and roots. In addition, the leaves are a place for photosynthate accumulation which is thought to contain elements of organic substances that have disease-curing properties, phenols, potassium compounds, and chlorophyll (Rusmina et al. 2015). The secondary metabolites from the leaves, such as alkaloids, saponins, and phenolic compounds, have been shown to have antimicrobial and antifungal effects, which may be capable for the pharmacological effects observed in the local communities. Nonetheless, certain diseases are treated with the whole plant. This accounts for $9 \%$ of the total plant species identified in this study. 
Table 2. List of plant species with medicinal values stated by the local community in Jambur Labu Village, Birem Bayeun Sub-district, East Aceh District, Aceh Province, Indonesia

\begin{tabular}{|c|c|c|c|c|c|c|}
\hline Scientific name & Family & $\begin{array}{c}\text { Vernacular } \\
\text { name }\end{array}$ & Part used & $\begin{array}{c}\text { Mode of } \\
\text { preparation }\end{array}$ & $\begin{array}{c}\text { Disease/ } \\
\text { ailment }\end{array}$ & $\begin{array}{c}\text { Use } \\
\text { value }\end{array}$ \\
\hline Acalypha indica $\mathrm{L}$. & Euphorbiaceae & Akar kucing & $\begin{array}{l}\text { Whole } \\
\text { plants }\end{array}$ & Decoction & Diarrhea & 0.16 \\
\hline Ageratum conyzoides L. & Asteraceae & Bandotan & Root & Pounded, smeared & Wounds & 0.33 \\
\hline Allamanda cathartica $\mathrm{L}$. & Apocynaceae & $\begin{array}{l}\text { Akar chempaka } \\
\text { hutan }\end{array}$ & Leaves & Pounded, affixed & Boils & 0.10 \\
\hline Allium fistulosum L. & Amaryllidaceae & Bawang kap & Leaves & Squeezed, affixed & Swollen & 0.13 \\
\hline Aloe vera (L.) Burm.f. & Asphodelaceae & Lidah buaya & Leaves & Pounded, affixed & Boils & 0.26 \\
\hline Alpinia galanga (L.) Willd. & Zingiberaceae & Laos & Rhizome & Pounded, smeared & Skin disorders & 0.73 \\
\hline Amaranthus spinosus L. & Amaranthaceae & Bayam eri & $\begin{array}{l}\text { Whole } \\
\text { plants }\end{array}$ & Pounded, smeared & Boils & 0.20 \\
\hline Anacardium occidentale $\mathrm{L}$. & Anacardiaceae & Jambu mente & Leaves & Decoction & Sore skin & 0.36 \\
\hline Ananas comosus (L.) Merr. & Bromeliaceae & Nanas & Fruit & Raw consumption & Constipation & 0.10 \\
\hline Andrographis paniculata (Burm.F.) Nees & Acanthaceae & Takila & Leaves & Raw consumption & Diabetes & 0.03 \\
\hline Annona muricata $\mathrm{L}$ & Annonaceae & Nangka sabrang & Fruit & Raw consumption & Gallstones & 0.03 \\
\hline Annona squamosa $\mathrm{L}$. & Annonaceae & Srikaya & Leaves & Pounded, affixed & Boils & 0.13 \\
\hline Apium graveolens $\mathrm{L}$. & Apiaceae & Seledri & $\begin{array}{l}\text { Whole } \\
\text { plants }\end{array}$ & Raw consumption & Rheumatism & 0.06 \\
\hline Archidendron jiringa (Jack) I.C.Nielsen & Fabaceae & Jengkol & Fruit & Eaten as vegetable & Flatulence & 0.26 \\
\hline Areca catechu L. & Arecaceae & Pinang & & Raw consumption & Oral hygiene & 0.26 \\
\hline Artocarpus altilis (Parkinson) Fosberg. & Moraceae & Sukun & Leaves & Decoction & Uric acid & 0.13 \\
\hline Artocarpus heterophyllus Lam. & Moraceae & Nangko & Fruit & Pounded, smeared & Antiacne & 0.23 \\
\hline Averrhoa bilimbi L. & Oxalidaceae & Blimbing wuluh & Fruit & Pounded, affixed & Mumps & 0.20 \\
\hline Averrhoa carambola $\mathrm{L}$. & Oxalidaceae & Blimbing legi & Fruit & Decoction & Cough & 0.40 \\
\hline Bidens pilosa $\mathrm{L}$. & Asteraceae & Ketul & Root & Dropped & Sore eyes & 0.06 \\
\hline Bougainvillea spectabilis Willd. & Nyctaginaceae & Bugenvil & Flower & Pounded, smeared & Boils & 0.30 \\
\hline Breynia androgyna (L.) Chakrab.\& N.P.Balakr. & Phyllanthaceae & Katukan & Leaves & Eaten as vegetable & Breast milk & 0.96 \\
\hline Cananga odorata (Lam.) Hook.f. \& Thomson & Annonaceae & Kenongo & Fruit & Decoction & Bronchitis & 0.50 \\
\hline Canna indica $\mathrm{L}$. & Cannaceae & Ganyong & Seed & Pounded, smeared & Headache & 0.13 \\
\hline Capsicum аппиит $\mathrm{L}$. & Solanaceae & Lombok jemprit & Fruit & Pounded, smeared & Hair growth & 0.06 \\
\hline Carica papaya $\mathrm{L}$ & Caricaceae & Kates & Fruit & Raw consumption & Flatulence & 0.83 \\
\hline Catharanthus roseus (L.) G.Don & Apocynaceae & Tapak doro & Leaves & Decoction & Diabetes & 0.06 \\
\hline Centella asiatica (L.) Urb. & Apiaceae & Pacul goang & $\begin{array}{l}\text { Whole } \\
\text { plants }\end{array}$ & Pounded, affixed & Boils & 0.30 \\
\hline Cibotium barometz (L.) J.Sm. & Cyatheaceae & Paku simpai & Rhizome & Decoction & Rheumati & 0.06 \\
\hline Citrus hystrix DC. & Rutaceaea & Jeruk purut & Leaves & Decoction & Fatigue & 0.66 \\
\hline Citrus L. & Rutaceaea & Limau kuit & & Squeezed & Sprue & 0.06 \\
\hline Citrus $x$ aurantiifolia (Christm.) Swingle & Rutaceaea & Jeruk pecel & & Squeezed & Cough & 0.73 \\
\hline Citrus $x$ aurantium $\mathrm{L}$. & Rutaceaea & & & Raw consumption & Constipation & 0.23 \\
\hline .) Osbeck & Rut: & & & leezed & Sore $\mathrm{t}$ & 0.30 \\
\hline Cocos nuci & Are & & & sumption & Heat in & 0.93 \\
\hline Colocasia esculenta (L.) Schoot & Arac & Tales & & Decoction & Stomach & 0.03 \\
\hline Cucurbita pepo L. & Cucurbitaceae & Labu & Fruit & Eaten as vegetable & Anthelmintic & 0.03 \\
\hline Curcuma longa $\mathrm{L}$. & Zingiberaceae & Kunir & Rhizome & Decoction & Typhus & 0.93 \\
\hline Curcuma mangga Valeton \& Zijp. & Zingiberaceae & Temu poh & Rhizome & Decoction & Menstrual laxative & 0.73 \\
\hline Cymbopogon citratus (DC.) Stapf. & Poaceae & Sereh dapur & $\begin{array}{l}\text { Whole } \\
\text { plants }\end{array}$ & Decoction & Cough & 0.13 \\
\hline Cymbopogon winterianus Jowitt ex Bor & Poaceae & Sere wangi & Rhizome & Decoction & Cough & 0.70 \\
\hline Datura metel L. & Solanaceae & Kecubung & Leaves & Pounded, affixed & Dermatitis & 0.33 \\
\hline Dimocarpus aristatus (Blume) Miq. & Sapindaceae & Kelengkeng & Fruit & Raw consumption & $\begin{array}{l}\text { Digestive } \\
\text { problems }\end{array}$ & 0.06 \\
\hline Dracaena trifasciata (Prain) Mabb. & Asparagaceae & Lidah mertua & & Decoction & Hemorrhoid & 0.06 \\
\hline Echinacea purpurea (L.) Moench & Asteraceae & $\begin{array}{l}\text { Kembang } \\
\text { kertas }\end{array}$ & $\begin{array}{l}\text { Whole } \\
\text { plants }\end{array}$ & Pounded, affixed & Fever & 0.06 \\
\hline Elephantopus scaber L. & Asteraceae & Tapak tangang & Root & Decoction & & 0.26 \\
\hline Euphorbia hirta L. & Euphorbiaceae & Kukon-kukon & $\begin{array}{l}\text { Whole } \\
\text { plants }\end{array}$ & Decoction & Asthma & 0.33 \\
\hline Euphorbia tithymaloides L. & Euphorbiaceae & & Sap & & & 0.26 \\
\hline Garcinia mangostana $\mathrm{L}$ & Clusiaceae & Manggis & Skin & Decoction & Sprue & 0.06 \\
\hline Gardenia jasminoides J.Ellis & Rubiaceae & Ceplong piring & Leaves & Pounded, affixed & Sprain & 0.23 \\
\hline Gerbera jamesonii Bolus & Asteraceae & Gerbera & Leaves & Decoction & Calm the nerves & 0.20 \\
\hline Gomphrena globosa L. & Amaranthaceae & Bunga kancing & Flower & Decoction & Asthma & 0.06 \\
\hline
\end{tabular}


Graptophyllum pictum (L.) Griff Hippobroma longiflora (L.) G.Don

Impatiens balsamina $\mathrm{L}$. Ipomoea aquatica Forssk. Ipomoea batatas (L.) Lam. Ixora javanica (Blume) DC. Jasminum sambac (L.) Aiton Jatropha curcas L.

Jatropha multifida L.

Kaempferia galanga L.

Kalanchoe pinnata (Lam.) Pers.

Lansium domesticum Correa

Mangifera indica L.

Manihot esculenta Crantz

Manilkara zapota (L.) P.Royen

Melastoma malabathricum $\mathrm{L}$.

Mirabilis jalapa $\mathrm{L}$.

Morinda citrifolia $\mathrm{L}$.

Moringa oleifera Lam.

Murraya koenigii (L.) Spreng.

Musa x paradisiaca $\mathrm{L}$.

Mussaenda frondosa $\mathrm{L}$.

Nephelium lappaceum L.

Ocimum tenuiflorum $\mathrm{L}$.

Orthosiphon aristatus (Blume) Miq

Pandanus amaryllifolius Roxb. ex Lindl.

Peperomia pellucida (L.) Kunth

Persea americana Mill.

Phaleria macrocarpa (Scheff.) Boerl.

Phragmanthera capitata (Spreng.) Balle

Phyllanthus niruri L.

Piper aduncum L.

Piper betle $\mathrm{L}$.

Piper crocatum Ruiz \& Pav.

Piper nigrum $\mathrm{L}$.

Polyscias scutellaria (Burm.f.) Fosberg

Psidium guajava $\mathrm{L}$.

Ricinus communis $\mathrm{L}$

Saccharum officinarum L.

Senna siamea (Lam.) H.S.Irwin \& Barneby

Sisyrinchium palmifolium $\mathrm{L}$.

Solanum stramoniifolium Jacq.

Spondias dulcis Parkinson

Strychnos lucida $\mathrm{R} . \mathrm{Br}$

Syzygium aqueum (Burm.f.) Alston)

Syzygium malaccense (L.) Merr. \& L.M.Perry

Syzygium polyanthum (Wight) Walp.

Tagetes erecta L.

Tamarindus indica $\mathrm{L}$.

Theobroma cacao $\mathrm{L}$.

Tithonia diversifolia (Hemsl.) A. Gray

Tradescantia spathacea $\mathrm{Sw}$.

Wollastonia biflora (L.) DC.

\begin{tabular}{|c|c|c|c|c|c|}
\hline Acanthaceae & Demung & Leaves & Decoction & Hemorrhoids & 0.13 \\
\hline Campanulaceae & Kitolod & $\begin{array}{l}\text { Whole } \\
\text { plants }\end{array}$ & Dripped & $\begin{array}{l}\text { Eye } \\
\text { inflammations }\end{array}$ & 0.47 \\
\hline Balsaminaceae & Pacar banyu & Leaves & Pounded, affixed & Snakebite & 0.03 \\
\hline Convolvulaceae & Kangkung & Stem & Eaten as vegetable & Constipation & 0.23 \\
\hline Convolvulaceae & Ketela rambat & Leaves & Pounded, smeared & Boils & 0.13 \\
\hline Rubiaceae & Soka & Leaves & Pounded, affixed & Sprains & 0.16 \\
\hline Oleaceae & Melati & Leaves & Decoction & Fever & 0.36 \\
\hline Euphorbiaceae & Jarak pager & Leaves & Affixed & Flatulence & 0.93 \\
\hline Euphorbiaceae & Betadine & Sap & Dripped & Wounds & 0.96 \\
\hline Zingiberaceae & Kencur & Rhizome & Pounded, smeared & Sprain & 0.63 \\
\hline Crassulaceae & Teres & Leaves & Pounded, smeared & Fever & 0.06 \\
\hline Meliaceae & Duku & Skin & Pounded & Fever & 0.20 \\
\hline Anacardiaceae & Mangga & Fruit & Raw consumption & Sprue & 0.16 \\
\hline Euphorbiaceae & Ubi kayu & Leaves & Pounded, smeared & Rheumatism & 0.06 \\
\hline Sapotaceae & Sawo & Fruit & Raw consumption & Dysentery & 0.13 \\
\hline Melastomataceae & Senggani & Root & Decoction & Hemorrhoids & 0.20 \\
\hline Nyctaginaceae & $\begin{array}{l}\text { Kembang pagi } \\
\text { sore }\end{array}$ & Leaves & Pounded, smeared & Boils & 0.16 \\
\hline Rubiaceae & Pace & Fruit & Squeezed & Hypertension & 0.13 \\
\hline Moringaceae & Kelor & Leaves & Pounded, smeared & Jaundice & 0.96 \\
\hline Rutaceae & Salam koja & Leaves & Decoction & Anemia & 0.30 \\
\hline Musaceae & Gedang & Fruit & Raw consumption & Jaundice & 0.06 \\
\hline Rubiaceae & Nusa indah putih & Leaves & Pounded, smeared & Boils & 0.20 \\
\hline Sapindaceae & Rambutan & Skin & Decoction & Dysentery & 0.03 \\
\hline Lamiaceae & Kemangi & Leaves & Eaten as vegetable & Fever & 0.43 \\
\hline Lamiaceae & Remujung & Root & Decoction & Uric acid & 0.06 \\
\hline Pandanaceae & Pandan & Leaves & Heated, rubbed & Sore & 0.26 \\
\hline Piperaceae & Suruhan & $\begin{array}{l}\text { Whole } \\
\text { plants }\end{array}$ & Decoction & Stomach ache & 0.06 \\
\hline Lauraceae & Alpokat & Leaves & Decoction & Hypertension & 0.06 \\
\hline Thymelaeaceae & Makuto rojo & Leaves & Pounded, smeared & Wound & 0.50 \\
\hline Loranthaceae & $\begin{array}{l}\text { Kembang } \\
\text { sepatu }\end{array}$ & Leaves & Decoction & Malaria & 0.06 \\
\hline Phyllanthaceae & Meniran & Root & Decoction & $\begin{array}{l}\text { Kidney } \\
\text { medicine }\end{array}$ & 0.23 \\
\hline Piperaceae & Sirihan & Sap & Smeared & Boils & 0.83 \\
\hline Piperaceae & Sedah & Leaves & Pounded, affixed & Tooth ache & 0.66 \\
\hline Piperaceae & Sirih merah & Leaves & Decoction & Nosebleed & 0.66 \\
\hline Piperaceae & Marica & Leaves & Decoction & Asthma & 0.30 \\
\hline Araliaceae & $\begin{array}{l}\text { Godong } \\
\text { mangkokan }\end{array}$ & Leaves & Pounded, smeared & Wounds & 0.60 \\
\hline Myrtaceae & Jambu klutuk & Leaves & Squeezed & Diarrhea & 0.46 \\
\hline Euphorbiaceae & Jarak kaliki & Leaves & Affixed & Digestive problems & 0.30 \\
\hline Poaceae & Rosan & Stem & Squeezed & Cough & 0.26 \\
\hline Fabaceae & Johar & Root & Decoction & Diabetes & 0.10 \\
\hline Iridaceae & Teki sabrang & Tubers & Decoction & Jaundice & 0.10 \\
\hline Solanaceae & Cokowana & Root & Decoction & Back pain & 0.06 \\
\hline Anacardiaceae & Kedondong & Leaves & Decoction & Cough & 0.16 \\
\hline Loganiaceae & Dara laut & Skin & Decoction & Rheumatism & 0.33 \\
\hline Myrtaceae & Jambu air & Fruit & Raw consumption & Sprue & 0.83 \\
\hline Myrtaceae & Jambu bold & Fruit & Raw consumption & Sprue & 0.53 \\
\hline Myrtaceae & Salam & Leaves & Decoction & Uric acid & 0.40 \\
\hline Asteraceae & $\begin{array}{l}\text { Bunga taik } \\
\text { lincong }\end{array}$ & Flower & Pounded, affixed & Colds & 0.96 \\
\hline Fabaceae & Asam jawa & Fruit & Decoction & Sprue & 0.26 \\
\hline Malvaceae & Cokelat & Fruit & Raw consumption & $\begin{array}{l}\text { Lower blood } \\
\text { pressure }\end{array}$ & 0.10 \\
\hline Asteraceae & Rondo noleh & Leaves & Decoction & Stomach ache & 0.56 \\
\hline Commelinaceae & Nanas kerang & Leaves & Pounded, smeared & Sprains & 0.30 \\
\hline Asteraceae & Widelia & $\begin{array}{l}\text { Whole } \\
\text { plants }\end{array}$ & Pounded, smeared & Pus sores & 0.03 \\
\hline Zingiberaceae & Bengle & Rhizome & Pounded, smeared & Joint pain & 0.96 \\
\hline Zingiberaceae & $\mathrm{Jae}$ & Rhizome & Decoction & Body warmer & 0.96 \\
\hline
\end{tabular}


The local people frequently used various plants in the study area to treat minor ailments such as coughs, fevers, and wounds. Citrus aurantiifolia fruit, for example, is mixed with honey to treat coughs, while Jasminum sambac leaves are used to treat fever. This study is similar to Enejoh et al. (2015), who found a community in Nigeria used C. aurantifolia fruit juice mixed with sugar, palm oil, or honey to treat cough. $C$. aurantifolia contains a wide range of bioactive compounds, including flavonoids (Wang et al. 2007), which provide a strong inherent potential to modify the body's response to allergens, viruses, and carcinogens (Okwu 2005). Local tribes in the study area also used Cymbopogon citratus to treat coughs. $C$. citratus has been traditionally used by people worldwide to treat various diseases such as cough, carminative, expectorant, and depurative (Avoseh et al. 2015).

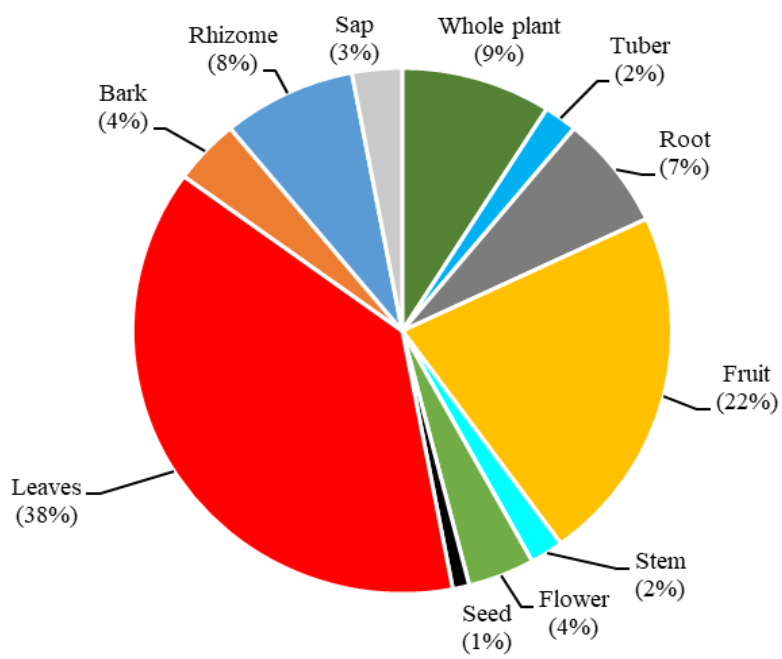

Figure 2. Parts of the medicinal plant species used by the local community in Jambur Labu Village, Birem Bayeun Sub-district, East Aceh District, Aceh Province, Indonesia

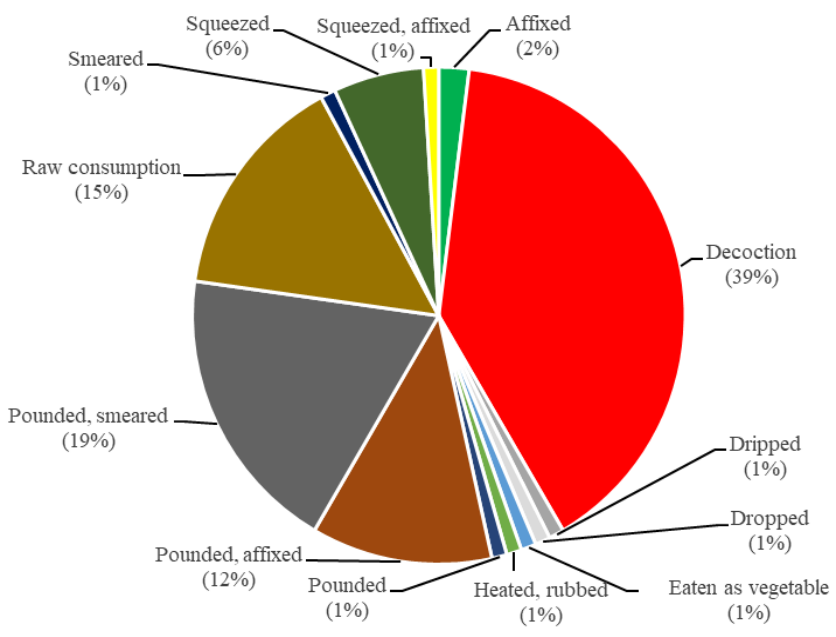

Figure 3. Mode of preparation of the medicinal plants used by the local community in Jambur Labu Village, Birem Bayeun Subdistrict, East Aceh District, Aceh Province, Indonesia

\section{Mode of preparation}

There were several modes of preparation of the medicinal plants used by the community surveyed in this study (Figure 3). The people of Jambur Labu usually processed the leaves by boiling them to drink the water. Other reports by Qasrin et al. (2020) found that the Malay tribal community of Lingga Regency, Riau Islands preferred the method of processing medicinal plants by boiling, soaking, pounding, brewing, squeezing, and without processing. Meanwhile, Rusmina et al. (2015) also found that the Mandar tribe in Sarude Sarjo village, North Mamuju District, West Sulawesi, used medicinal plants by drinking boiled water with a percentage of $43 \%$. Treatment by drinking after boiling is known to have better efficacy than treatment by drinking without boiling.

\section{Use-Value of medicinal plant species}

The result of the calculation showed that the use-values (UV) of medicinal plants used by the Jambur Labu community ranged from 0.03 to 0.96 (Table 2). Breynia androgyna, Moringa oleifera, Jatropha multifida, Zingiber montanum, Zingiber officinale, and Tagetes erecta had the highest UV value (0.96). Plants with a high UV value are medicinal plants that have many benefits and are recognized by most respondents. In contrast, plants with low UV values are plants that have little or no efficacy recognized by respondents. This is in line with BeltránRodríguez et al. (2014) which states that the use-value can be used to determine the level of traditional knowledge of local communities. The value of using plants as medicine is strongly influenced by culture, the spiritual beliefs of local communities (Cocks and Dold 2006), and geography (Pieroni 2001).

\section{Informant consensus factor}

The diseases mentioned by the informants were first classified according to the International Disease Classification. From these categories, ICF values were calculated (Table 3).

A total of 56 types of diseases were found in Jambur Labu Village which can be grouped into 18 categories (Table 3). The values of the Informant Consensus Factor (ICF) ranged from as low as 0.948 to as high as 0.978 . ICF values determine the consistency of the utilisation of a plant species for a certain disease. The mental and behavioral disorders (MBD) and the diseases of the blood and blood-forming organs and certain disorders involving the immune mechanism (DBF) groups had the highest ICF value (0.978). The Diseases of the Digestive System had the second-highest ICF (0.976). Higher values indicated that the respondents only used a few plant species to treat a particular disease, whereas low values indicated low agreement on which plant to use for a specific disease. Anemia, fatigue, and toothache are common diseases among the people of the study area. In relation to adequate nutritional intake, anemia is more common in pregnant mothers and young women (Yadav et al. 2017; Sinawangwulan et al. 2018). 
Table 3. Category of ailment and the Informant Consensus Factor (ICF) stated by local community in Jambur Labu Village, Birem Bayeun Sub-district, East Aceh District, Aceh Province, Indonesia

\begin{tabular}{|c|c|c|c|}
\hline Ailment category & Specified disease name & $\begin{array}{l}\text { No. of use } \\
\text { reports }\end{array}$ & ICF value \\
\hline Certain infectious and parasitic diseases (CID) & $\begin{array}{l}\text { Dysentery, diarrhea, mumps, wound, malaria, } \\
\text { kidney medicine, sore, body warmer, skin } \\
\text { disorders, typhus }\end{array}$ & 592 & 0.967 \\
\hline Certain infectious and parasitic diseases (CIP) & Swollen, anthelmintic & 367 & 0.969 \\
\hline Diseases of the circulatory system (CSD) & Hypertension & 67 & 0.954 \\
\hline $\begin{array}{l}\text { Certain conditions originating in the perinatal } \\
\text { period (COP) }\end{array}$ & Breast milk & 203 & 0.965 \\
\hline $\begin{array}{l}\text { Diseases of the blood and blood-forming organs } \\
\text { and certain disorders involving the immune } \\
\text { mechanism (DBF) }\end{array}$ & Anemia & 185 & 0.978 \\
\hline Diseases of the digestive system (DSD) & $\begin{array}{l}\text { Hemorrhoids, constipation, sprue, hepatitis, } \\
\text { hemorrhoid, gallstones }\end{array}$ & 172 & 0.976 \\
\hline Diseases of the skin and subcutaneous tissue (DS) & Skin pain, dermatitis, scabies, anti-acne, boils & 78 & 0.961 \\
\hline Diseases of the eye and adnexa (EAD) & Hypertension, eye inflammation & 159 & 0.949 \\
\hline External causes of morbidity and mortality (EM) & Uric acid, digestive problems, flatulence, hot inside & 488 & 0.969 \\
\hline $\begin{array}{l}\text { Endocrine, nutritional and metabolic diseases } \\
\text { (ENM) }\end{array}$ & Diabetes, nosebleed, digestive problems & 116 & 0.956 \\
\hline $\begin{array}{l}\text { Factors influencing health status and contact with } \\
\text { health services (FHS) }\end{array}$ & Menstrual pain & 61 & 0.966 \\
\hline $\begin{array}{l}\text { Injury, poisoning, and certain other consequences } \\
\text { of external causes (IPD) }\end{array}$ & Snakebite, sprain, lowering blood pressure, sprain & 78 & 0.948 \\
\hline Mental and behavioral disorders (MBD) & Fatigue, toothache & 47 & 0.978 \\
\hline $\begin{array}{l}\text { Diseases of the musculoskeletal system and } \\
\text { connective tissue (MCD) }\end{array}$ & Joint pain, rheumatism & 74 & 0.972 \\
\hline Neoplasms (N) & Calms the nerves, hair growth, digestive problems & 222 & 0.972 \\
\hline Diseases of the nervous system (NSD) & Sprain & 166 & 0.975 \\
\hline Diseases of the respiratory system (RSD) & Cough, bronchitis, sore throat, asthma & 416 & 0.966 \\
\hline $\begin{array}{l}\text { Symptoms, signs, and abnormal clinical and } \\
\text { laboratory (SSA) }\end{array}$ & Fever, jaundice, headache, cough & 436 & 0.963 \\
\hline
\end{tabular}

Traditional knowledge about medicinal plants has been passed down from generation to generation in the local community in the study area. During the discussion, respondents stated that many children were interested in learning about medicinal plants from traditional healers or elders in their village. This study consistent with Suwardi et al. (2021) reported that traditional knowledge education on the use of medicinal plants has been given to children in the Aneuk Jamee tribe from an early age.

Finally, the large number of medicinal plants cited by local communities illustrated valuable local knowledge in Jambur Labu Village. The findings revealed that local communities in Jambur Labu Village used 107 medicinal plant species from 87 families for medicinal purposes to treat 56 different diseases, with anemia, fatigue, and toothache being the most widely treated. Breynia androgyna, Moringa oleifera, Jatropha multifida, Zingiber montanum, Zingiber officinale, and Tagetes erecta were the most commonly used medicinal plants by local communities, with a use-value index of 0.96. Local communities recognized the leaf $(38 \%)$ as the most commonly used plant part. The five most common plant families used by local communities were reported to be Euphorbiaceae, Rutaceae, Zingiberaceae, Piperaceae, and Myrtaceae. Furthermore, the traditional knowledge must be preserved by involving the Indonesian government through the Education Office and incorporating traditional knowledge into the basic education curriculum. This activity could be used to conserve traditional knowledge, natural resources, and biodiversity.

\section{ACKNOWLEDGEMENTS}

We are grateful to all people in the Jambur Labu Village for their kind hospitality and sharing knowledge. We also thank the University of Samudra for supporting this study.

\section{REFERENCES}

Adam M, Ahmed AA, Yagi A, Yagi S. 2020. Ethnobotanical investigation on medicinal plants used against human ailments in Erkowit and Sinkat areas, Eastern Sudan. Biodiversitas 21 (7): 3255-3262. DOI: 10.13057/biodiv/d210748.

Avoseh O, Oyedeji O, Rungqu P, Nkeh-Chungag B, Oyedeji A. 2015. Cymbopogon Species: Ethnopharmacology, phytochemistry and the pharmacological importance. Molecules 20: 7438-7453. DOI: 10.3390/molecules20057438.

Azmin N, Rahmawati A, Hidayatullah ME. 2019. Uji kandungan fitokimia dan etnobotani tumbuhan obat tradisional berbasis pengetahuan lokal di Kecematan Lambitu Kabupaten Bima. Florea 
Jurnal Biologi dan Pembelajarannya 6 (2): 101-113. DOI: 10.25273/florea.v6i2.4678. [Indonesian]

Batoro J, Siswanto D. 2017. Ethnomedicinal survey of plants used by local society in Poncokusumo district, Malang, East Java Province, Indonesia. Asian J Med Biol Res 3 (2): 158-167. DOI: 10.3329/ajmbr.v3i2.33563.

Beltrán-Rodríguez L, Ortiz-Sánchez A, Mariano NA, MaldonadoAlmanza B, Reyes-García V. 2014. Factors affecting ethnobotanical knowledge in a mestizo community of the Sierra de Huautla Biosphere Reserve, Mexico. J Ethnobiol Ethnomed 10: 14. DOI: 10.1186/1746-4269-10-14

Calzada F, Bautista E. 2020. Plants used for the treatment of diarrhoea from Mexican flora with amoebicidal and giadicidal activity, and their phytochemical constituents. J Ethnopharmacol 253: 112676. DOI: 10.1016/j.jep.2020.112676.

Cocks ML, Dold AP. 2006. Cultural significance of biodiversity: The role of medicine plant in urban African cultural practices in the Eastern Cape, South Africa. J Ethnobiol 26 (1): 69-78. DOI: 10.2993/02780771(2006)26[60:CSOBTR]2.0.CO;2.

Eldeen IMS, Effendy MAW, Muhammad TST. 2016. Ethnobotany: Challenges and future perspectives. Res J Med Plants 10: 382-387. DOI: $10.3923 /$ rjmp.2016.382.387.

Elfahmi, Woerdenbag H, Kayser O. 2014. Jamu: Indonesian traditional herbal medicine towards rational phytopharmacological use. J Herbal Med 4 (2): 51-73. DOI: 10.1016/j.hermed.2014.01.002.

Elfrida, Mubarak A, Suwardi AB. 2020. The fruit plant species diversity in the home gardens and their contribution to the livelihood of communities in rural area. Biodiversitas 21 (8): 3670-3675. DOI: 10.13057/biodiv/d210833.

Enejoh OS, Ogunyemi IO, Bala MS, Oruene IS, Suleiman MM, Ambali SF. 2015. Ethnomedical importance of Citrus Aurantifolia (Christm) Swingle. Pharm Innov J 4 (8): 1-6.

Hasanuddin, Djufri, Kusyanti, Nazaruddin. 2021. Ethnobotanical survey of medical plant used for the treatment of hypertension in Subulussalam sub-District Aceh, Indonesia. Jurnal Biotik 9 (1): 1-14 [Indonesian]

Jadid N, Kurnaiawan E, Himayani CES, Andriyani, Prasetyowati I, Purwani KI, Muslihatin W, Hidayati D, Tjahjaningrum ITD. 2020. An ethnobotanical study of medicinal plantsused by the Tengger tribe in Ngadisari village, Indonesia. PLoS One 15 (7): e0235886. DOI 10.1371/journal.pone.0235886.

Malini DM, Madihah, Kusmoro J, Kamilawati F, Iskandar J. 2017. Ethnobotanical study of medicinal plants in Karangwangi, District of Cianjur, West Java. Biosaintifika 9 (2): 345-356. DOI 10.15294/biosaintifika.v9i2.5756.

Mustofa FI, Rahmawati N, Aminullah. 2020. Medicinal plants and practices of Rongkong Traditional Healers in South Sulawesi, Indonesia. Biodiversitas 21 (2): 642-651. DOI: $10.13057 /$ biodiv/d210229.

Navia ZI, Chikmawati T. 2015. Durio tanjungpurensis (Malvaceae), a new species and its one new variety from West Kalimantan, Indonesia. Bangladesh J Bot 44 (3): 429-436. DOI: 10.3329/bjb.v44i3.38550

Navia ZI, Suwardi AB, Harmawan T, Syamsuardi, Mukhtar E. 2020a. The diversity and contribution of indigenous edible fruit plants to the rural community in the Gayo Highlands, Indonesia. J Agric Rural Dev Trop Subtrop 121 (1): 89-98. DOI:10.17170/kobra-202004061145.

Navia ZI, Audira D, Afifah N, Turnip K, Nuraini, Suwardi AB. 2020b. Ethnobotanical investigation of spice and condiment plants used by the Taming tribe in Aceh, Indonesia. Biodiversitas 21 (10): 4467 4473. DOI: $10.13057 /$ biodiv/d211001.

Navia ZI, Suwardi AB, Nuraini. 2021. The importance of tropical edible fruit plants for tribal communities in East Aceh region, Indonesia IOP Conf Series: Earth Environ Sci 637 (1): 012003. DOI 10.1088/1755-1315/637/1/012003

Okwu DE. 2005. Phytochemicals, vitamins and mineral contents of two nigerian medicinal plants. Intl J Mol Med Adv Sci 1: 375-381.

Pieroni A. 2001. Evaluation of the cultural significance of wild food botanical traditionally comsumed in Northwestern Tuscany, Italy. J Ethnobiol 21 (1): 89-104.
Qasrin U, Setiawan A, Bintoro A. 2020. Study on ethnobotany of medicinal plants used by the Malay Tribe, Lingga district, Kepulauan Riau. Jurnal Belantara 3 (2): 139-152. DOI: 10.29303/jbl.v3i2.507. [Indonesian]

Rahmawati N, Mustofa FI, Haryanti S. 2020. Diversity of medicinal plants utilized by To Manui ethnic of Central Sulawesi, Indonesia. Biodiversitas 21 (1): 375-392. DOI: 10.13057/biodiv/d210145

Rosita SMD, Rostiana O, Pribadi ER, Hernani. 2007. Penggalian iptek etnomedisin di Gunung Gede Pangrango. Buletin Penelitian Tanaman Rempah dan Obat 18 (1): 13-28. [Indonesian]

Rusmina HZ, Miswan, Pitopang R. 2015. Studi etnobotani tumbuhan obat pada masyarakat Suku Mandar di Desa Sarude Sarjo Kabupaten Mamuju Utara Sulawesi Barat. Jurnal Biocelebes 9 (1): 73-87. [Indonesian]

Silalahi M, Supriatna J, Walujo EB, Nisyawati. 2015. Local knowledge of medicinal plants in sub-ethnic Batak Simalungun of North Sumatra, $\begin{array}{llll}\text { Indonesia. } & \text { Biodiversitas } 16 & \text { (1): 44-54. DOI: }\end{array}$ 10.13057/biodiv/d160106

Sinawangwulan IP, Dewi YLR, Wekadigunawan CSP. 2018. Association between Socio-demographic, Nutrition Intake, Cultural Belief, and Incidence of Anemia in Pregnant Women in Karanganyar, Central Java. Journal of Maternal and Child Health 3 (2): 128-137. DOI: 10.26911/thejmch.2018.03.02.05

Sujarwo W, Keim AP, Savo V, Guarrera PM, Caneva G. 2015. Ethnobotanical study of Loloh: Traditional herbal drinks from Bali (Indonesia). J Ethnopharmacol 169: 34-48. DOI: 10.1016/j.jep.2015.03.079

Sutrisno IH, Bachtiar A, Navia ZI, Nuraini, Suwardi AB, 2020. Documentation of ritual plants used among the Aceh tribe in Peureulak, East Aceh District, Indonesia. Biodiversitas 21 (22): 49904998. DOI: $10.13057 /$ biodiv/d211102

Suwardi AB, Navia ZI, Harmawan T, Syamsuardi, Mukhtar, E. 2019. The diversity of wild edible fruit plants and traditional knowledge in West Aceh region, Indonesia. J Med Plants 7 (4): 285-290. DOI: 10.1088/1757-899X/725/1/012064

Suwardi AB, Navia ZI, Harmawan T, Nuraini, Syamsuardi, Mukhtar E. 2020a. Ethnobotany, nutritional composition and sensory evaluation of Garcinia from Aceh, Indonesia. IOP Conf Ser Mater Sei Eng 725 (1): 012064. DOI: 10.1088/1757-899X/725/1/012064.

Suwardi AB, Navia ZI, Harmawan T, Syamsuardi, Mukhtar E. 2020b. Wild edible fruits generate substantial income for local people of the Gunung Leuser National Park, Aceh Tamiang region. Ethnobot Res Appl 20: 1-13. DOI:10.32859/era.20.11.1-13.

Suwardi AB, Navia ZI, Harmawan T, Syamsuardi, Mukhtar E. 2020c. Ethnobotany and conservation of indigenous edible fruit plants in South Aceh, Indonesia. Biodiversitas 21 (5): 1850-1860. DOI: 10.13057/biodiv/d210511

Suwardi AB, Mardudi, Navia ZI, Baihaqi, Muntaha. 2021. Documentation of medicinal plants used by Aneuk Jamee tribe in Kota Bahagia Subdistrict, South Aceh, Indonesia. Biodiversitas 22 (1): 2085-4722. DOI: 10.13057/biodiv/d220102.

The Central Bureau of Statistics of East Aceh District. 2020. Birem Bayeun Sub-district in figure 2019. The Central Bureau of Statistics of East Aceh district, Indonesia.

Wang YC, Chuang YC, Ku YH. 2007. Quantitation of bioactive compounds in citrus fruits cultivated in Taiwan. Food Chem 102 (4): 1163-1171. DOI: 10.1016/j.foodchem.2006.06.057.

Weckmüller H, Barriocanal C, Maneja R. 2019. Factors Afecting Traditional Medicinal Plant Knowledge of the Waorani, Ecuador. Sustainability 11: 4460. DOI: 10.3390/su11164460.

Yadav J, Yadav AK, Srinadh R. 2017. Rural-urban disparities in prevalence of anemia among adolescent girls in India. Int $\mathrm{J}$ Community Med Public Health. 4 (12): 4661-4667. DOI: DOI: 10.18203/2394-6040.ijcmph20175347.

Yeung AWK, Heinrich M, Kijjoa A, Tzvetkov NT, Atanasov AG. 2020. The ethnopharmacological literature: An analysis of the scientific landscape. J Ethnopharmacol 250: 112414. DOI: 10.1016/j.jep.2019.112414. 